\title{
Colonização de substrato artificial por macroinvertebrados límnicos, no delta do rio Jacuí (RS, Brasil)
}

\author{
Daniel Pereira ${ }^{1 *}$ \\ Maria Cristina Dreher Mansur ${ }^{1}$ \\ Cecília Volkmer-Ribeiro ${ }^{2}$ \\ Márcia Divina de Oliveira ${ }^{3}$ \\ Cíntia Pinheiro dos Santos ${ }^{1}$ \\ Paulo Eduardo Aydos Bergonci ${ }^{1}$ \\ Centro de Ecologia, Universidade Federal do Rio Grande do Sul \\ Avenida Bento Gonçalves, 9500, Setor 4, CEP 91540-000, Porto Alegre - RS, Brasil \\ ${ }^{2}$ Museu de Ciências Naturais da Fundação Zoobotânica do Rio Grande do Sul \\ ${ }^{3}$ Empresa Brasileira de Pesquisa Agropecuária, Centro de Pesquisa Agropecuária do Pantanal \\ *Autor para correspondência \\ dani.mdourado@gmail.com
}

Submetido para publicação em 29/07/2009

Aceito para publicação em09/10/2009

\section{Resumo}

Com a finalidade de avaliar a colonização de um substrato artificial por macroinvertebrados límnicos, foram realizados experimentos em duas estações de coleta no delta do rio Jacuí, em Porto Alegre (RS, Brasil): Canal do Jacuí (CJ) e Cais do Porto (PO). Os substratos foram constituídos de garrafa PET com uma malha interna de nylon. Os organismos que apresentaram os maiores valores de densidade média no substrato artificial exposto no CJ foram L. fortunei (8229,0ind. $\left.\mathrm{m}^{-2}\right)$, Chironomidae $\left(188,9 \mathrm{ind}_{\mathrm{m}} \mathrm{m}^{-2}\right)$ e Heleobia piscium $\left(39,6 \mathrm{ind} . \mathrm{m}^{-2}\right)$ e no PO, L. fortunei $\left(3233,0\right.$ ind $\left.\mathrm{m}^{-2}\right)$, Chironomidae $\left(288,9 \mathrm{ind}_{\mathrm{m}} \mathrm{m}^{-2}\right)$ e Oligochaeta $\left(211,1 \mathrm{ind} . \mathrm{m}^{-2}\right)$. Trochospongilla paulula apresentou uma área de cobertura de bioincrustação de $0,016 \mathrm{~m}^{2}$, equivalente a $31,1 \%$, da superfície externa do substrato (PET). A esponja de água doce, Trochospongilla paulula, também cresceu sobre as valvas dos mexilhões (epizoísmo). Por meio do teste $T$ de Student foram verificadas diferenças significativas $(p=0,01)$ entre os valores de densidade média de Oligochaeta verificados no CJ (menor valor) e PO (maior valor). Também foram verificadas diferenças altamente significativas $(\mathrm{p}<0,0001)$ entre as áreas de bioincrustação de T. paulula verificados no CJ (maior valor) e PO (menor valor). Os valores de densidade média dos demais taxa nos substratos expostos no CJ e PO não diferiram estatisticamente. $\mathrm{O}$ substrato foi adequado para amostrar macroinvertebrados, sobretudo poríferos, grupo de difícil amostragem.

Unitermos: bioinscrustação, biomonitoramento, epizoísmo, Limnoperna fortunei, Trochospongilla paulula

\section{Abstract}

Colonization of artificial substrate by freshwater macroinvertebrates, in the Jacuí River Delta, Rio Grande do Sul State, Brazil. Experiments to evaluate the limnic macroinvertebrates that colonize artificial 
substrates were performed at two experimental stations located in Jacui Channel (CJ) and Cais do Porto (PO), both part of the Jacui Delta in Porto Alegre, RS, southern Brazil. Substrates were built in PET bottles containing an internal nylon screen. In CJ, L. fortunei (8229.0 ind. $\left.\mathrm{m}^{-2}\right)$, Chironomidae (188.9 ind. $\mathrm{m}^{-2}$ ), and Heleobia piscium (39.6 ind. $\mathrm{m}^{-2}$ ) presented the highest values of average density. In PO, L. fortunei (3233.0 ind. $\mathrm{m}^{-2}$ ), Chironomidae (288.9 ind $\mathrm{m}^{-2}$ ), and Oligochaeta $\left(211.1\right.$ ind $\mathrm{m}^{-2}$ ) presented the highest values of average density. The freshwater sponge, Trochospongilla paulula, presented an incrustation area of $0.016 \mathrm{~m}^{2}$, that corresponded to $31 \%$ of the total external substrate surface (PET bottles). Trochospongilla paulula also grew over the golden mussel valves (epizoism). Significant differences between average densities of Oligochaeta verified in the sustrates from CJ (lowest value) and PO (highest value) were found using the statistics Mann-Whitney Test $(\mathrm{p}=0.01)$. Other significant differences $(\mathrm{p}<0.0001)$ between the bioincrustation areas of $T$. paulula in the substrates from CJ (highest value) and PO (lowest value) were found. The average densities of other taxa in substrates from both CJ and PO did not differ statistically. The substrate was adequate for the sampling of macroinvertebrates, especially Porifera, a group that is difficult to sample.

Key words: bioincrustation, biomonitoring, epizoism, Limnoperna fortunei, Trochospongilla paulula

\section{Introdução}

Os substratos artificiais são ferramentas amplamente utilizadas no monitoramento de macroinvertebrados límnicos e marinhos, especialmente de espécies que se fixam ou formam incrustações sobre superfícies duras. Os substratos artificiais padronizam a amostragem, reduzem a variabilidade e o tempo de processamento de amostras (Gibbons et al., 1993).

Volkmer-Ribeiro et al. (2004) desenvolveram um substrato artificial utilizando garrafas PET preenchidas com o retículo celulósico da fruta da bucha ( $L u f f a$ cylindrica L.), visando simular as raízes do aguapé de baraço, Eichhornia azurea Sw. (Kunth.), anteriormente demonstradas como substratos naturais de incrustação por fauna de macroinvertebrados bentônicos (VolkmerRibeiro et al., 1984). O dispositivo PET criado visava amostrar e monitorar populações de macroinvertebrados bentônicos no rio Caí e no seu contribuinte, arroio Bom Jardim, na área de influência do Pólo Petroquímico do Sul. Queiroz et al. (2007), desenvolveram um substrato artificial, com base na adaptação do dispositivo proposto por Volkmer-Ribeiro et al. (2004), substituindo o retículo da fruta da bucha por folhas de taboa (Typha dominguensis Pers.). Ambos os substratos desenvolvidos permitem a amostragem quantitativa de macroinvertebrados límnicos, sendo os resultados expressos como abundância dos organismos no substrato.

Até a década de 1990, a bioincrustação em ecossistemas límnicos de água doce da América do Sul era pouco representativa, salvo aquelas formadas pelas espécies do filo Porifera. No entanto, com a introdução do mexilhão dourado, Limnoperna fortunei (Dunker, 1857), na bacia do lago Guaíba, RS, um novo cenário se estabeleceu na macrofauna bentônica desta bacia. O mexilhão dourado produz fios de bisso que possibilitam a incrustação do mesmo sobre diferentes tipos de substratos duros formando macroaglomerados. Segundo Darrigran et al. (1998), associados aos macroaglomerados, se verifica uma fauna de macroinvertebrados rica e diversificada.

O monitoramento do mexilhão dourado tem sido realizado via a utilização de substratos artificiais com área conhecida, construídos em madeira (Oliveira et al., 2006; Takeda et al., 2007; Mansur et al., 2009) e em material cerâmico (Terra et al., 2007; Santos et al., 2008; Bergonci et al., 2009). Esses substratos permitem a amostragem quantitativa, sendo os resultados expressos como densidade (ind. $\mathrm{m}^{-2}$ ) dos organismos no substrato.

Uma série de experimentos visando avaliar a eficiência de substratos artificiais na colonização do mexilhão dourado e de macroinvertebrados límnicos foi realizada durante a execução do projeto de pesquisa "Programa de Pesquisa para Controle do Mexilhão Dourado (Limnoperna fortunei) nas Águas Jurisdicionais Brasileiras", coordenado pelo Instituto de Pesquisas do Mar da Marinha do Brasil (IEAPM), cujos resultados foram publicados parcialmente em (Mansur et al., 2009; Bergonci et al., 2009; Fernandes et al., 2007). O presente trabalho apresenta parte dos dados obtidos nos experimentos de colonização de macroinvertebrados 
límnicos em substrato artificial adaptado de VolkmerRibeiro et al. (2004) e Queiroz et al. (2007), no delta do rio Jacuí (RS, Brasil).

\section{Material e Métodos}

A área de estudo está situada no delta do rio Jacuí (Figura 1), na região metropolitana de Porto Alegre. Dentre os canais que atravessam esse delta se destaca o do Jacuí. Uma das estações de coleta onde foram submersos os substratos artificiais está situada na margem direita do Canal do Jacuí (CJ), com profundidade média de $8 \mathrm{~m}$, junto à Ilha da Pintada. A outra estação de coleta está situada na doca junto ao Corpo de Bombeiros, no Cais do Porto (PO). Neste local se verificam margens íngremes (paredes concretadas) e águas profundas $(5 \mathrm{~m})$.

O substrato (Figura 2c) foi construído a partir de uma adaptação do substrato artificial desenvolvido por Volkmer-Ribeiro et al. (2004) (Figura 2a) e do modelo adaptado do mesmo por Queiroz et al. (2007) (Figura 2b). Com o auxílio de uma tesoura, foi removido o fundo de uma garrafa PET; com um arame de aço foi fixada uma malha quadrada de nylon poliamida $\left(100 \mathrm{~cm}^{2} ;\right.$ malha de $1 \mathrm{~mm}$ de abertura) na lateral interior do recipiente; o fundo removido foi novamente fixado à garrafa com amarras de aço e, finalmente, foram feitos orifícios $(\varnothing$ $1 \mathrm{~cm}$ ) em toda a superfície do recipiente, com ferro em brasa. Os orifícios permitiriam a circulação da água e a entrada de organismos no interior do recipiente.

Os substratos permaneceram mergulhados por 90 dias nos meses de verão, época de maior produção larval do mexilhão dourado naquele ano (Soares et al., 2008; Mansur et al., 2009; Bergonci et al., 2009) e de maior diversidade e abundância da fauna bentônica e associada a substratos naturais constituídos de macrófitas aquáticas no delta do rio Jacuí, com base em dados históricos (Shröder-Pfeiffer e Pitoni, 2003; Tavares et al., 2005). O período de submersão do substrato artificial foi superior ao período máximo (75 dias) recomendado por Henriques de Oliveira (2002) para a avaliação da colonização de fauna bentônica em ecossistemas límnicos. Em dezembro (2005), foram implantadas nove réplicas do substrato artificial (Figura 2C), em cada estação de coleta, fixadas por meio de anilhas plásticas

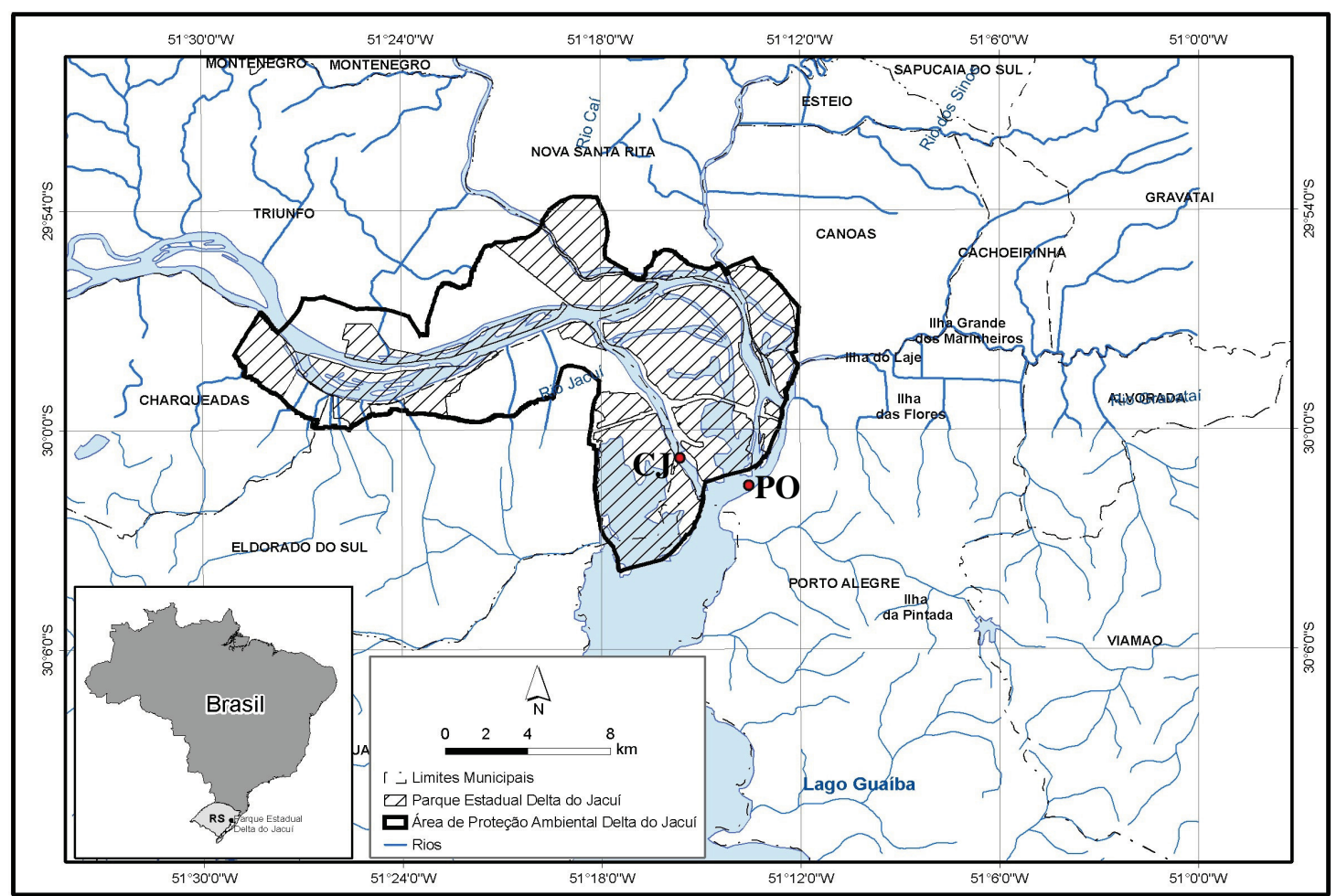

FIGURA 1: Estações de coleta no Canal do Jacuí (CJ) e Cais do Porto (PO), delta do rio Jacuí, Porto Alegre (RS, Brasil). A área central demarcada corresponde ao Parque Estadual Delta do Rio Jacuí. Mapa: Ricardo Aranha Ramos (MCN/FZB-RS). 


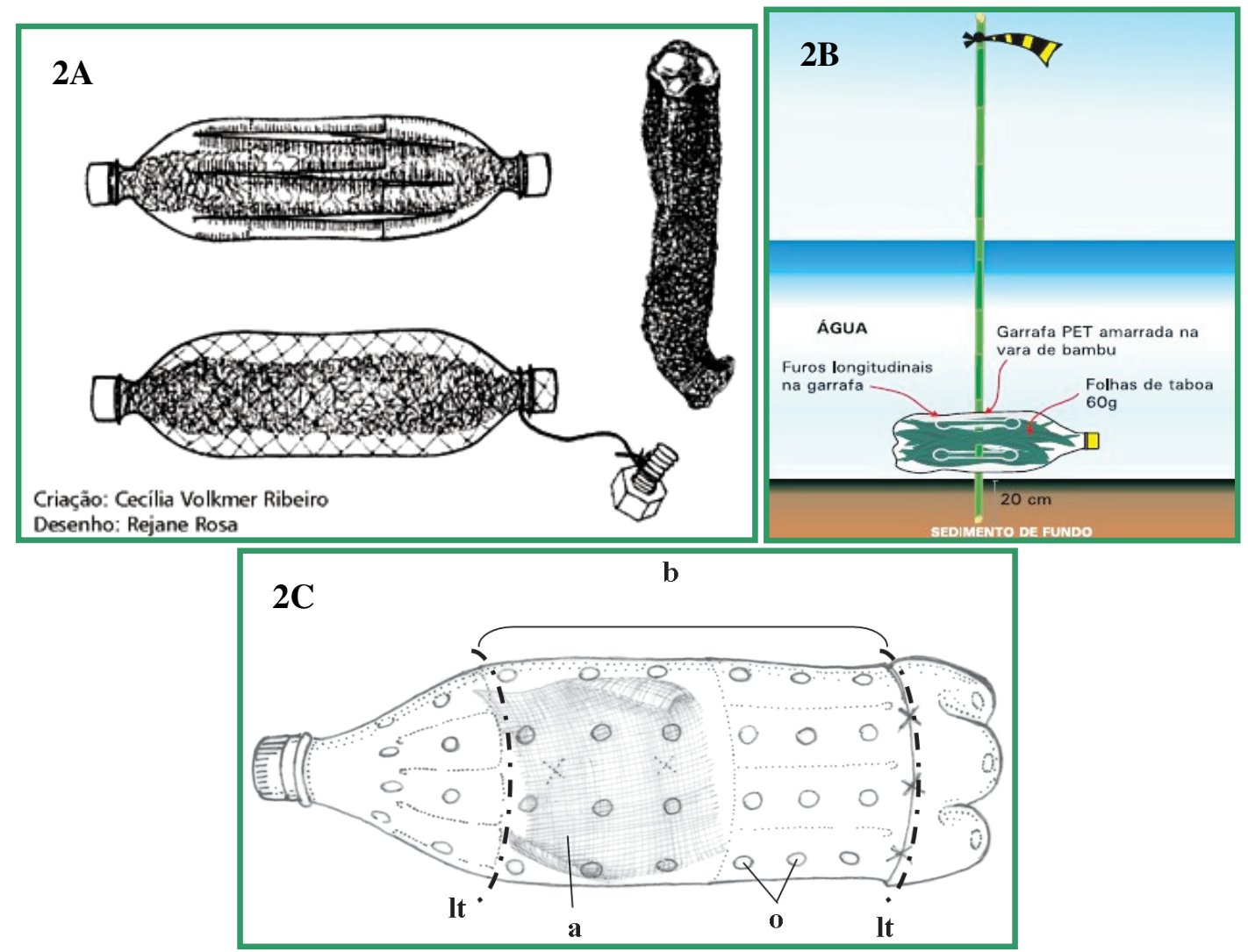

FIGURA 2: Substrato artificial para a colonização de macroinvertebrados límnicos: modelo original de Volkmer-Ribeiro et al. (2004) (2A); modelo adaptado de Volkmer-Ribeiro et al. (2004) por Queiroz et al. (2007) (2B); modelo proposto neste trabalho (2C): superfície amostral 1 (a), malha de nylon (área: $100 \mathrm{~cm}^{2}$ ) fixada na lateral interior da garrafa; superfície amostral 2 (b), retângulo (área: $576 \mathrm{~cm}^{2}$ ) formado pelo corte da lateral exterior da garrafa PET nas linhas tracejadas (lt); orifícios (o) com diâmetro de $1 \mathrm{~cm}$.

em uma grade de ferro submersa. No CJ a grade foi suspensa por cordas na parte inferior de um trapiche. Desta forma os substratos permaneceram igualmente mergulhados à coluna d'água na posição vertical. Já no PO a grade de ferro foi suspensa por cordas junto à parede concretada do cais. Em março de 2006, todos os substratos foram retirados para quantificação dos macroinvertebrados.

No laboratório, o fundo e o gargalo das garrafas foram removidos com o auxílio de uma tesoura. A malha de nylon (superfície amostral 1) foi removida e fixada em álcool 70\%. Foi realizada a quantificação total de macroinvertebrados sob o microscópio estereoscópico, sendo os resultados expressos em densidade média (ind. $\mathrm{m}^{-2}$ ). A malha de nylon utilizada no presente substrato foi um material inerte substituto aos materiais orgânicos, retículo celulósico da fruta de L. cylindrica e folhas de $T$. dominguensis, utilizados respectivamente por Volkmer-
Ribeiro et al. (2004) e por Queiroz et al. (2007).

Após a retirada da malha, a lateral da garrafa foi aberta (Figura 2B), originando um retângulo de $576 \mathrm{~cm}^{2}$ (superfície amostral 2). O material fixado na superfície externa foi quantificado sob o microscópio estereoscópico, sendo a cobertura da bioincrustação de esponjas expressa em a área $\left(\mathrm{m}^{2}\right)$ e percentual de cobertura (\%) e os macroinvertebrados expressos em densidade média (ind. $\mathrm{m}^{2}$ ).

Parte do material zoológico, indivíduos de $L$. fortunei e macroinvertebrados associados, foi depositada na coleção de moluscos do Museu de Ciências e Tecnologia da PUCRS: BRASIL, Rio Grande do Sul, Porto Alegre: Ilha da Pintada, delta do rio Jacuí, D.Pereira, M.C.D. Mansur, C.P. dos Santos e R.M.Z. Richinitti leg. (MCP 9117, 921 exs.); Cais do Porto, doca do corpo de bombeiros, D.Pereira, M.C.D. Mansur, C.P. dos Santos e R.M.Z. Richinitti leg. (MCP 9119, 16 
exs.). Os exemplares de poríferos foram depositados na coleção de poríferos de água-doce do Museu de Ciências Naturais da Fundação Zoobotânica do Rio Grande do Sul: BRASIL, Rio Grande do Sul, Porto Alegre Lago Guaíba, Cais do Porto, 29/03/2006, M.C.D. Mansur \& D. Pereira leg (MCN-POR 7786 a 7788; 7790 a 7796); Lago Guaíba, Cais do Porto, 11/04/2006, M.C.D. Mansur \& D. Pereira leg.(MCN-POR 7789).

Foi utilizado o teste $T$ de Student $(\alpha=0,05)$ para comparação entre os valores logaritimizados, Log $(\mathrm{x}+1)$, de densidades $(D)$ médias dos taxa de macroinvertebrados dos dois locais de coleta $\left(\mathrm{H}_{0}: D(\mathrm{CJ})\right.$ $=D(\mathrm{PO}))$. A ferramenta utilizada para a realização do teste foi o software SPSS 11.5.

\section{Resultados}

Após 90 dias de exposição dos substratos, foi verificada a colonização do substrato artificial por 10 taxa de macroinvertebrados límnicos. O número de taxa registrados no CJ foi sete e no PO, nove (Tabela 1). Na superfície amostral 1 (malha de nylon) foram registrados nove taxa e, na superfície amostral 2 (retângulo PET), apenas três taxa.

Os organismos que apresentaram os maiores valores de densidade média no substrato artificial colocados no CJ foram Limnoperna fortunei $\left(8229,0\right.$ ind. $\left.\mathrm{m}^{-2}\right)$, Chironomidae $\left(188,9 \mathrm{ind}_{\mathrm{m}} \mathrm{m}^{-2}\right)$ e Heleobia piscium $\left(39,6\right.$ ind $\left.\mathrm{m}^{-2}\right)$. No mesmo local, a superfície amostral 2 apresentou apenas dois taxa: L. fortunei $\left(862,3\right.$ ind. $\left.\mathrm{m}^{-2}\right) \mathrm{e}$ H. piscium $\left(17,4\right.$ ind. $\left.\mathrm{m}^{-2}\right)$. Já no PO, os organismos que apresentaram os maiores valores de densidade média no substrato artificial foram L. fortunei (3233ind. $\mathrm{m}^{-2}$ ), Chironomidae (288,9ind. $\left.\mathrm{m}^{-2}\right)$ e Oligochaeta $\left(211,1\right.$ ind. $\left.\mathrm{m}^{-2}\right)$. No mesmo local, a superfície amostral 2 apresentou apenas três taxa: a esponja de água doce Trochospongilla paulula (Bowerbank, 1863), com área de cobertura de bioincrustação de $0,016 \mathrm{~m}^{2}$, correspondente a $31,1 \%$ da área da superfície amostral 2 do substrato e, L. fortunei e $H$. piscium, com os respectivos valores de densidade média 862,3 e 211 ind. $\mathrm{m}^{-2}$.

Pequenas aglomerações de mexilhões e esponjas obstruíram alguns orifícios do substrato artificial (Figura 3). Além de utilizar a superfície do PET como substrato, T. paulula também utilizou as valvas dos mexilhões (epizoísmo) que se fixaram juntos aos orifícios (Figura 4).

Por meio do teste $T$ (Tabela 1 ) foram verificadas diferenças significativas $(p=0,01)$ entre os valores de densidade média de Oligochaeta verificados nos substratos expostos no CJ (menor valor) e PO (maior valor). Também foram verificadas diferenças significativas $(p<0,0001)$ entre os valores de cobertura da bioincrustação de $T$. paulula verificados nos substratos expostos no CJ (maior valor) e $\mathrm{PO}$ (menor valor). Os valores de densidade média dos demais táxons nos substratos expostos no CJ e PO não diferiram estatisticamente.

TABELA 1: Densidade de táxons de macroinvertebrados límnicos, média terro padrão (mínimo-máximo), nas superfícies amostrais (SA) do substrato colonizado no delta do rio Jacuí (RS, Brasil). Diferenças significativas verificadas por meio do teste de T de Student $(*)$.

\begin{tabular}{|c|c|c|c|c|c|}
\hline Táxons & SA & Unidade & Canal do Jacuí (CJ) & Cais do Porto (PO) & Teste $\mathbf{T}$ \\
\hline $\begin{array}{l}\text { PORIFERA } \\
\text { Demospongiae } \\
\text { Spongillidae }\end{array}$ & & & & & \\
\hline Trochospongilla paulula & 2 & $\begin{array}{c}\mathrm{m}^{2} \\
\%\end{array}$ & $\begin{array}{l}0,0 \\
0,0\end{array}$ & $\begin{array}{c}0,016 \pm 0,003(0,0-0,024) \\
31,1 \pm 3,7(5,7-41,5)\end{array}$ & $\begin{array}{l}<0,0001^{*} \\
<0,0001^{*}\end{array}$ \\
\hline $\begin{array}{l}\text { ANELLIDA } \\
\text { Oligochaeta } \\
\text { Hirudinea }\end{array}$ & 1 & ind. $m^{-2}$ & $33,3 \pm 23,6(0,0-200,0)$ & $211,1 \pm 73,5(0,0-600,0)$ & $0,01 *$ \\
\hline $\begin{array}{l}\text { Glossiphonidae } \\
\text { MOLLUSCA } \\
\text { Gastropoda } \\
\text { Sorbeoconcha }\end{array}$ & 1 & ind. $\mathrm{m}^{-2}$ & 0,0 & $22,2 \pm 22,2(0,0-200,0)$ & 0,33 \\
\hline
\end{tabular}




\section{Cochliopidae}

Heleobia piscium

$$
\begin{array}{ccc}
1 & \text { ind. } \mathrm{m}^{-2} & 22,2 \pm 14,7(0,0-100,0) \\
2 & \text { ind.m } & 17,4 \pm 7,7(0,0-52,1) \\
1+2 & \text { ind. } m^{-2} & 39,6 \pm 13,4(0,0-100,0)
\end{array}
$$

$100,0 \pm 50,0(0,0-300,0)$

0,46

$10,3 \pm 6,5(0,0-52,1)$

0,15

Basomatophora

$104,5 \pm 49,0(0,0-300,0)$

0,09

\section{Ancylidae}

Burnupia sp.

Bivalvia

Mytiloida

\section{Mytilidae}

$\begin{array}{cccc}\text { Limnoperna fortunei } & 1 & \text { ind. } \mathrm{m}^{-2} & 7366,7 \pm 2167,4 \\ & & (0,0-20100,0) \\ & 2 & \text { ind. } \mathrm{m}^{-2} & 862,3 \pm 258,3 \\ & & (17,4-2048,6) \\ & 1+2 & \text { ind. } \mathrm{m}^{-2} & 8229,0 \pm 2284,7 \\ & & & (652,1-21280,6)\end{array}$

$$
\begin{gathered}
2766,7 \pm 1555,7 \\
(0,0-14000,0) \\
468,7 \pm 150,1 \\
(17,4-1232,6) \\
3233,0 \pm 1520,3 \\
(225,69-14017,4)
\end{gathered}
$$

INSECTA

Trichoptera

\section{Leptoceridae}

Hydropsichidae

$$
\text { ind. } \mathrm{m}^{-2} \quad 22,2 \pm 14,7(0,0-100,0)
$$

0,0

0,15

Diptera

\begin{tabular}{lllcrl} 
Chironomidae & 1 & ind. $\mathrm{m}^{-2}$ & $188,9 \pm 117,2$ & $288,9 \pm 206,5(0,0-1900,0)$ & 0,88 \\
Ephydridae & 1 & ind. $\mathrm{m}^{-2}$ & $0,0 \pm 0,0(0,0-0,0)$ & $33,3 \pm 33,3(0,0-300,0)$ & 0,33 \\
\hline
\end{tabular}

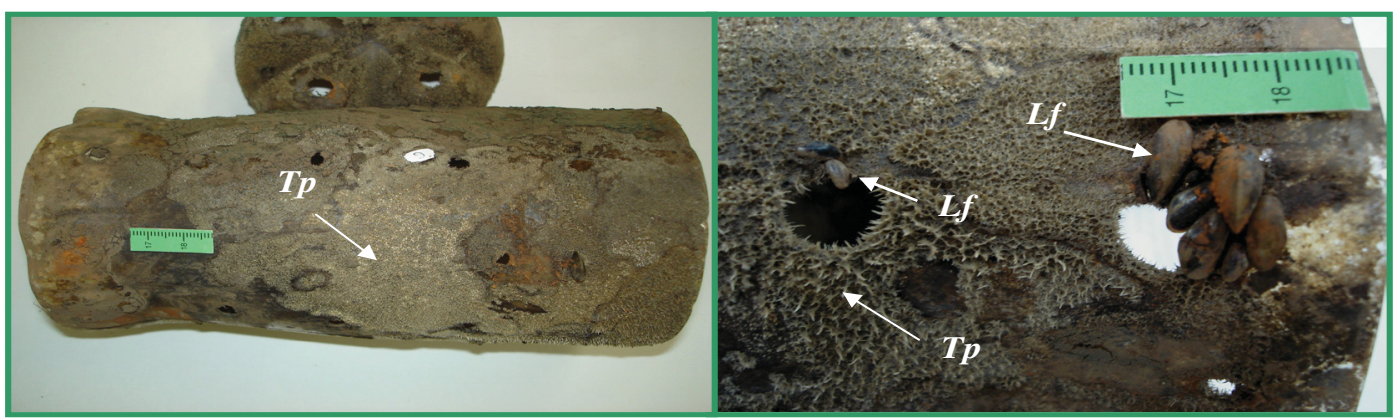

FIGURA 3: Superfície amostral 2 incrustada pela esponja de água doce Trochospongilla paulula (Bowerbank, 1863) (Tp) e por Limnoperna fortunei (Dunker, 1857) (Lf). Fotos: Vanessa de Souza Machado.

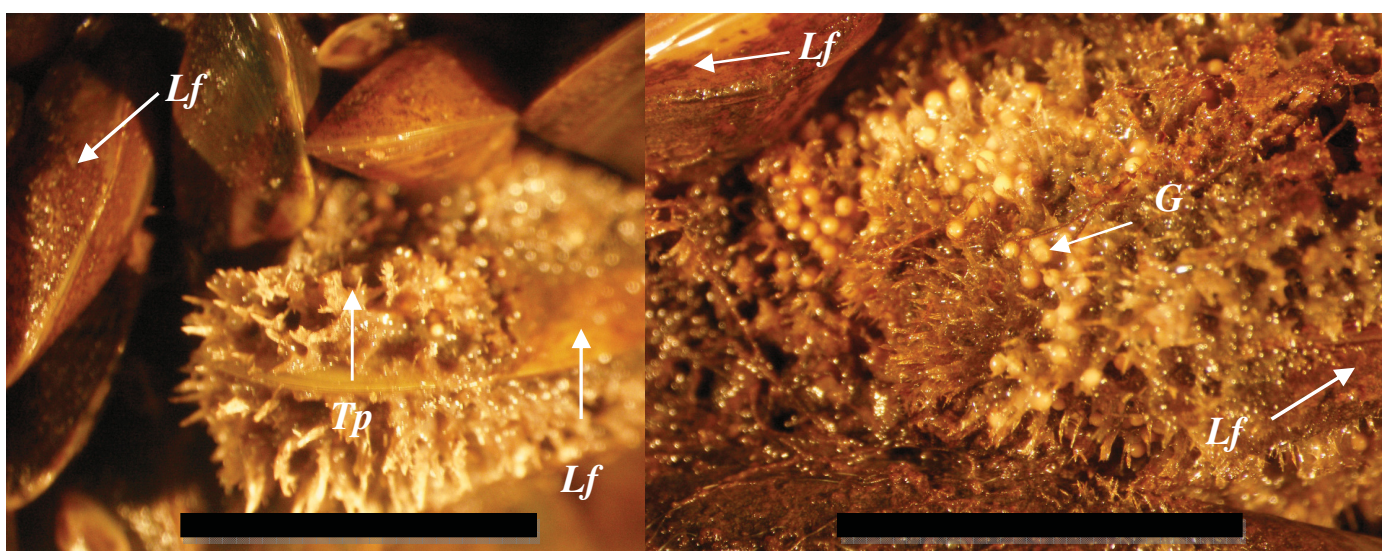

FIGURA 4: Epizoísmo: Trochospongilla paulula (Bowerbank, 1863) (Tp) colonizando e crescendo sobre a superfície das conchas de indivíduos de Limnoperna fortunei (Dunker, 1857) (Lf) retirados do substrato artificial. Fotos: Maria Cristina Dreher Mansur. Gêmulas da esponja (G). Escala: $1 \mathrm{~cm}$. 


\section{Discussão}

Comparando o número de táxons verificado no presente trabalho com os números encontrados por Volkmer-Ribeiro et al. (2004) e Queiroz et al. (2007), podemos fazer algumas considerações. O substrato adaptado por Queiroz et al. (2007) foi submerso em viveiros de tilápia ricos em matéria orgânica e em nutrientes, ou seja, com qualidade da água similar àquela verificada na área estudada no delta do rio Jacuí, enquanto que, o substrato desenvolvido por Volkmer-Ribeiro et al. (2004) foi instalado em rios de água limpa.

Comparando os menores valores de riqueza de taxa de macroinvertebrados verificados no substrato testado no delta do Jacuí (10 taxa), com os valores obtidos por Volkmer-Ribeiro et al. (2004) no curso inferior do rio Caí (68 taxa), cabe ressaltar que no delta os substratos foram suspensos na coluna d'água, enquanto que no rio Caí os substratos foram fixados junto ao leito. A suspensão do substrato na coluna d'água teve, provavelmente, como via de colonização principal, os macroinvertebrados e suas respectivas larvas que estavam à deriva no plâncton. No segundo caso, configurou-se elevado potencial de migração de organismos já colonizados no leito e margens do rio, resultando em abundância e riqueza mais elevada. Outro fator a ser considerado é que a malha de nylon apresenta menor área amostral do que o retículo de L. cylindrica, com câmaras interiores capazes de abrigar diferentes grupos animais e sustentar comunidade perifítica (alimento para raspadores). Além disso, o próprio retículo constitui uma fonte de alimento para organismos fragmentadores e detritívoros. Sobre o material celulósico da bucha de L. cylindrica se desenvolve comunidade perifítica que atua na ciclagem do fósforo e nitrogênio disponível na água (Nabisadeh et al., 2008; Souza et al., 2008; Oboh et al., 2009), assim como dos sólidos retidos e suas câmaras internas, o que constitui em disponibilidade de alimento a comunidade bentônica.

No presente estudo se destacaram, em termos de abundância, L. fortunei, Chironomidae e Oligochaeta, e de área de cobertura, T. paulula. Dentre os taxa verificados por Volkmer-Ribeiro et al. (2004) após 60 dias de exposição dos substratos artificiais, destacaram- se com maior abundância: Turbellaria, $H$. piscium, Oligochaeta, Cyclopidae, Podocopida, Lepidoptera, Coleoptera e Bryozoa. Após 75 dias de exposição dos substratos artificiais com preenchimento de folhas de T. domingensis, em viveiros de produção de tilápia, Queiroz et al. (2007) verificaram a colonização de 15 taxa de macroinvertebrados límnicos, número muito próximo ao verificado no presente estudo. Os autores verificaram a predominância, em termos de abundância, de moluscos gastrópodes invasores Thiaridae, de sanguessugas Glossiphonidae e dípteros Chironomidae, em águas enriquecidas com nutrientes provenientes da ração utilizada na alimentação da tilápia.

Ambos os materiais não inertes (L. cylindrica $\mathrm{e}$ Typha domingensis) sofrem modificações ao longo do tempo de exposição que podem influenciar a sucessão e colonização dos macroinvertebrados límnicos. Segundo Álvarez e Bécares (2006), a decomposição de Typha latifolia apresenta diferenças significativas entre as estações do ano quanto à perda de massa vegetal e concentração de nitrogênio. Por outro lado, o conteúdo do substrato testado, malha de nylon comercial (mistura de nylon 66, nylon 1010 e poliamida), apresenta alta resistência à abrasão, resistência química, aos solventes e óleos, elevada dureza e rigidez (IDES, 1986-2009). Desta forma constitui um substrato mais estável que possibilita uma melhor padronização da amostragem.

Além de utilizar a superfície do PET como substrato, T. paulula também utilizou as valvas dos mexilhões (epizoísmo) que se fixaram juntos aos orifícios. Pequenas aglomerações de mexilhões e esponjas obstruíram alguns orifícios do substrato artificial (Figura 3). Trata-se do primeiro registro para o Brasil desta associação entre a esponja e o mexilhão dourado. Segundo Ezcurra de Drago (2004), L. fortunei ocupa e compete, desde a sua fase plantígrada bissada, pelos mesmos substratos que as esponjas de água doce, podendo ocorrer também a competição por alimento. A autora registrou pela primeira vez na Argentina, um aglomerado de mexilhão dourado crescendo entre o mesohilo de uma esponja de água doce do gênero Trochospongilla Vejdovsky, 1888 incrustada sobre um tronco de Solanum glaucophyllum. Além de uma possível competição pode haver também uma associação com benefícios para ambas as espécies 
filtradoras e bioincrustantes. Sugere-se para estudos futuros melhor examinar a relações ecológicas interespecíficas entre $L$.fortunei e $T$. paulula com ênfase na competição por espaço e por alimento.

Segundo Tavares et al. (2003), T. paulula é comum nos macrofitais do delta do rio Jacuí, e particularmente abundante incrustando as raízes de Eichhornia crassipes (Mart.) Solms-Laubach. e E. azurea. A esponja produz gêmulas durante todo ano, mas apresenta maior abundância no outono (Tavares et al., 2005), no Saco do Ferraz. Justamente este local, com elevada produção de gêmulas, está localizado a montante do PO com sua abertura direcionada para o mesmo, o que explica a incidência deste porífero nessa estação de ancoragem dos substratos artificiais. Deve-se ainda tomar em consideração que o substrato artificial testado, especialmente a superfície amostral 2 (limpa e dura), instalado a jusante do fluxo dispersor das gêmulas produzidas nos sacos da ilha, foi o ideal para a fixação dessas gêmulas.

A inexistência de incrustações de esponjas nos substratos artificiais colocadas no CJ pode ser atribuída ao aporte maior de sedimento neste canal. Assim como a deposição de sedimento, também foi verificado visualmente a colonização de perifíton sobre a superfície amostral 2 do substrato artificial. Tais fatores constituíram impeditivo para a fixação de gêmulas de $T$. paulula, que são escassas neste local.

As diferenças estatísticas verificadas quanto às densidades de Oligochaeta refletem as condições da qualidade da água. No PO, onde os Oligochaeta foram mais densos, foi verificado sedimento organicamente enriquecido junto à malha de nylon. Segundo Rosemberg e Resh (1993), estes organismos são indicadores de ambientes ricos em matéria orgânica. Também se deve ressaltar que a esponja formou bioincrustações muito rasas sobre os substratos artificiais e estas estavam carregadas de gêmulas, indicando um substrato favorável (Figura 4), mas uma qualidade de água desfavorável. Esta área do delta do Jacuí apresenta os mais elevados níveis de poluição (Faria e Lersch, 1998; Bendati et al., 2003).

Heleobia piscium é uma espécie de gastrópode muito comum na bacia do rio Jacuí e no lago Guaíba, ocorrendo em diferentes tipos de substratos em águas de arroios, canais de irrigação, rios, açudes e lagos, no sedimento de fundo e junto às macrófitas aquáticas (Pereira et al., 2000 e 2001; Mansur et al., 2009). A sua ampla distribuição em diferentes tipos de microhabitats nesta região explica a elevada incidência e densidade populacional no substrato avaliado.

Os valores de densidade média de $L$. fortunei no substrato artificial testado no delta do Jacuí são inferiores aos valores verificados em substrato artificial constituído de material de madeira (Mansur et al., 2009) e cerâmico (Bergonci et al., 2009) testados no mesmo delta. Limnoperna fortunei prefere substratos mais rígidos semelhantes aos substratos rochosos e galhos submersos de "sarandi" (Cephalantus glabratus (Spreng.), comuns no lago Guaíba e delta do rio Jacuí (Santos et al., 2008). Estudos realizados por outros autores quanto à predominância de taxa de macroinvertebrados associados ao mexilhão dourado demonstraram resultados similares aos constatados no substrato artificial no delta do Jacuí: Oligochaeta e Chironomidae (Fernandes et al., 2007; Freitas et al., 2008); Hirudinea (Darrigran et al., 1998); H. piscium (Darrigran et al., 1998; Mansur et al., 2008), e Trochospongilla sp. (Ezcurra de Drago, 2004).

O substrato artificial proposto, constituído de materiais inertes, com área conhecida, proporciona amostragens quantitativas que resultam em valores de densidade populacional de taxa de macroinvertebrados. Já os substratos constituídos de garrafas PET preenchidas com materiais orgânicos, avaliados por Volkmer-Ribeiro et al. (2004) e Queiroz et al. (2007), proporcionam amostragem semiquantitativa que resultam em valores de abundância de macroinvertebrados, ou seja, o número de organismos presentes no substrato.

O material inerte externo (PET) do substrato se demonstrou adequado à amostragem de poríferos, enquanto que a malha de nylon se demonstrou adequada aos demais organismos. Diferentemente dos outros substratos citados, com preenchimentos não inertes (L. cylindrica e T. dominguensis) o presente substrato oferece condições estáveis durante todo o período de colonização. Estes três tipos de substratos artificiais resultam em padrões de amostragem diferenciados que refletem as propriedades dos materiais com os quais foram construídos. A escolha apropriada de 
uma determinada ferramenta de amostragem deve levar em consideração a finalidade do programa de biomonitoramento, a composição específica da fauna alvo de amostragem, as características ambientais do ecossistema aquático e a seletividade do substrato artificial (Silveira e Queiroz, 2006).

\section{Agradecimentos}

Ao CNPq pelo auxílio concedido CNPq/CT-Hidro (processo n. 507675/2004-5, período 2005-2008) bem como bolsa de produtividade em pesquisa e respectivo Grant a C. Volkmer Ribeiro (Proc. 306266/2006-7).

\section{Referências}

Álvarez, J. A.; Bécares, E. 2006. Seasonal decomposition of Typha latifolia in a free-water surface constructed wetland. Ecological Engineering, 28 (2): 99-105.

Bendati, M. M. A.; Schwarzbach, M. S.; Maizonave, C. R. M.; Bringhenti, M. L.; Almeida, L. B. 2003. Avaliação da qualidade da água do Lago Guaíba: Subsíduos para a gestão da bacia hidrográfica. Ecos, 7: 1-34.

Bergonci, P. E. A.; Mansur, M. C. D.; Pereira, D.; Santos, C. P. 2009. Population sampling of the golden mussel, Limnoperna fortunei (Dunker, 1857), based on artificial ceramic substrate. Biotemas, 22 (3): 85-94.

Darrigran, G.; Martin, S. M.; Gullo, B.; Armendariz, L. 1998. Macroinvertebrados associated to the byssus of Limnoperna fortunei (Dunker, 1857) (Pelecipoda, Mytilidae). Río de la Plata, Argentina. Hydrobiología, 367: 223-230.

Ezcurra-de-Drago, I. 2004. Biodiversidad de Porifera en el Litoral Argentino. Grado de Competencia con el Bivalvo invasor Limnoperna fortunei (Dunker, 1857) (Bivalvia, Mytilidae). In: Aceñolaza, F. G. (Org.). Temas de la biodiversidad del litoral fluvial argentino. INSUGEO, Miscelanea, Argentina, p.195-204.

Faria, C. M.; Lersch, E. C. 1998. Delta do Jacuí: Águas e sedimento. DMAE, Porto Alegre, Brasil, 56pp.

Fernandes, S. E.; Takeda, A. M.; Fujita, D. S.; Pinha, G. D.; Behrend, R. D. L.; Ragonha, F. H. 2007. Substrato artificial em diferentes canais secundários da planície aluvial do alto rio Paraná: Limnoperna fortunei versus invertebrados aquáticos. Anais do II Encontro Sul-americano de Integração de Ações para o Controle do Mexilhão Dourado, Rio de Janeiro, Brasil, p.16.

Freitas, S. M. F.; Kapusta, S. C.; Santos, C. P.; Rodriguez, M. T. 2008. Indicadores bióticos: Macrofauna bentônica associada à Limnoperna fortunei (mexilhão-dourado). Anais do X Congresso Brasileiro de Ecotoxicologia, Porto Alegre, Brasil, p.283.

Gibbons, W. N.; Munn, M. D.; Paine, M. D. 1993. Guidelines for monitoring benthos in freshwater environments. B.C. by EVS Consultants, North Vancouver, Canadá, 81pp.

Henriques de Oliveira, C. 2002. Macroinvertebrados associados à Typha domingensis Pers (Typhaceae) em duas lagoas no litoral norte fluminense e sua utilização em programas de biomonitoramento. Dissertação de Mestrado, Universidade Federal do Rio de Janeiro, Brasil, 92pp.

IDES. 1986-2009. The plastic web: Polyamide (Nylon) Applications. Disponível em <http://www.ides.com/generics/ Nylon/Nylon_applications.htm>. Acesso em 27 de setembro de 2009.

Nabizadeh, R.; Naddafi, K.; Mesdaghinia, A.; Nafez, A. H. 2008. Feasibility study of organic matter and ammonium removal using loofa sponge as a supporting medium in an aerated submerged fixed-film reactor (ASFFR). Electronic Journal of Biotechnology, 11 (4): 1-9.

Mansur, M. C. D.; Santos, C. P.; Richinitti, L. M. Z.; Pereira, D.; Batista, C. B.; Alberto, R. M. F.; Silva, M. C. P. 2008. Ocorrência de moluscos límnicos e crustáceo em macroaglomerados do mexilhão dourado, Limnoperna fortunei (Dunker, 1857) sobre sarandi no lago Guaíba (RS, Brasil). Biotemas, 21 (4): 179-182.

Mansur, M. C. D.; Pereira, D.; Santos, C. P.; Bergonci, P. E. A.; Thormann, B. M.; Takeda, A. M. 2009. Colonização de substrato artificial de madeira pelo mexilhão dourado, Limnoperna fortunei (Dunker, 1857) (Bivalvia, Mytiloida, Mytilidae), no Delta do Rio Jacuí (RS, Brasil). Biotemas, 22 (1): 75-80.

Oliveira, M. D.; Takeda, A. M.; Barros, L. F.; Barbosa, D. S.; Resende, E. 2006. Invasion by Limnoperna fortunei (Dunker, 1857) (Bivalvia, Mytilidae) of Pantanal wetland, Brasil. Biological Invasions, 8: 97-104.

Oboh, I.; Aluyor, E.; Audu, T. 2009. Post-treatment of Produced water before discharge using Luffa cylindrica. Leonardo Electronic Journal of Practices and Technologies, 14: 57-64.

Pereira, D.; Consoni, J. M.; Inda, L. A.; Konrad, H. G. 2001. Composição e abundância de espécies de moluscos do bentos marginal da microbacia do arroio Capivara, Triunfo, RS, Brasil. Biociências, 9 (1): 3-20.

Pereira, D.; Veitenheimer-Mendes, I. L.; Mansur, M. C. D.; Silva, M. C. P. 2000. Malacofauna límnica do sistema de irrigação do arroio Capivara e áreas adjacentes, Triunfo, RS, Brasil. Biociências, 8 (1): $137-157$.

Queiroz, J. F.; Silveira, M. P.; Sitton, M.; Marigo, A. L. S.; Zambom, G. V.; Silva, J. R.; Carvalho, M. P.; Ribacinko, R. B. 2007. Coletor de macroinvertebrados bentônicos com substrato artificial para monitoramento da qualidade de água em viveiros de produção de tilápia. Circular Técnica - EMBRAPA Meio Ambiente, 16: 1-5.

Rosemberg, D. N; Resch.,V. H. 1993. Freshwater biomonitoring and benthic macroinvertebrates. Chapman \& Hall, New York, USA, 488pp.

Santos, C. P.; Würdig, N. L.; Mansur, M. C. D. 2008. Variações no comprimento dos indivíduos de uma população do mexilhão dourado, Limnoperna fortunei (Mollusca: Bivalvia: Mytilidae), ao longo do ano, na Praia do Veludo, lago Guaíba, Rio Grande do Sul, Brasil. Revista Brasileira de Zoologia, 25 (3): 389-396.

Schröder-Pfeifer, N. T.; Lopes-Pitoni, V. L. 2003. Análise qualitativa estacional da fauna de moluscos límnicos no delta do Jacuí, RS, Brasil. Biociências, 11 (2): 145-158.

Silveira, M. P.; Queiroz, J. F. 2006. Uso de coletores com substrato artificial para monitoramento biológico de qualidade de água. Comunicado Técnico EMBRAPA, 39: 1-5. 
Soares, M. F; Pires, M. J.; Mansur, M. C. D.; Pereira, D.; Santos, C. P. 2008. Desenvolvimento e adequação de tecnologias ambientais para o controle populacional do mexilhão dourado Limnoperna fortunei. CNPq/FEEVALE/PUCRS, Novo Hamburgo, Brasil, 99pp. Disponível em <http://cadastrocthidro.ana.gov.br/arquivos/ relatorio_cnpq.pdf $>$.

Acesso em 9 de julho de 2009.

Sousa, J. T.; Henrique, I. N.; Oliveira, R.; Lopes, W. S.; Valderi Leite, V. D. 2008. Nitrification in a submerged attached growth bioreactor using Luffa cylindrica as solid substrate. African Journal of Biotechnology, 7 (15): 2702-2706.

Takeda, A. M.; Fujita, D. S.; Fontes Jr, H. M. 2007. Bivalves invasores no rio Paraná. In: Santos, S. B.; Pimenta, A. D.; Thiengo, S. C.; Fernandez, M. A.; Absalão, R. S. (Orgs). Tópicos em Malacologia: Ecos do XVIII EBRAM. Sociedade Brasileira de Malacologia, Rio de Janeiro, Brasil, p.81-86.

Tavares, M. C. M.; Volkmer-Ribeiro, C.; De Rosa-Barbosa, R. 2003. Primeiro registro de Corvoheteromeyenia australis (Bonetto \& Ezcurra de Drago) para o Brasil com chave taxonômica para os poriferos do Parque Estadual Delta do Jacuí, Rio Grande do Sul, Brasil. Revista Brasileira de Zoologia, 20 (2): 169-182.

Tavares, M. C. M.; Volkmer-Ribeiro, C.; Hermany, G. 2005. Seasonal abundance in a sponge assembly at a Southern Neotropical Inner Delta. Journal of Coastal Research, 42: 335-342.

Terra, N. R.; Lemieszek, M. B.; Lemos, C. T.; Leite, E. H. 2007. Presença de Limnoperna fortunei (Dunker, 1857) nas bacias hidrográficas do Uruguai e lago Guaíba, Rio Grande do Sul, Brasil. FEPAM em Revista, 1 (1): 12-19.

Volkmer-Ribeiro, C.; Guadagnin, D. L.; De Rosa-Barbosa, R.; Silva, M. M.; Drügg-Hahn, S.; Lopes-Pitoni, V. L.; Gastal, H. A. O.; Barros, M. P.; Demaman, L. V. 2004. A Polyethylenetherephthalate (PET) device for sampling freshwater benthic macroinvertebrates. Brazilian Journal of Biology, 64 (3A): 531-541.

Volkmer-Ribeiro, C.; Mothes de Moraes, B.; De Rosa-Barbosa, R.; Mansur, M. C. D.; Veitenheimer-Mendes, I. L. 1984 Um estudo do Bentos em raízes de Eichhornia azurea (Sw.) Kunth, do curso inferior de um rio subtropical sul-americano. Revista Brasileira de Biologia, 44 (2): 125-132. 